\title{
Generalization of the Dewar Formula for Total Energies of Molecules
}

\author{
V. GINEITYTE \\ Institute of Theoretical Physics and Astronomy, Gostauto 12, 2600 Vilnius, Lithuania
}

Received 11 January 1999; revised 31 July 1999; accepted 31 August 1999

\begin{abstract}
An expression for the total energies of molecules $E$ was derived using the power series for the one-electron density matrix (DM) $P$ obtained previously in the basis of bond orbitals (BOs) [Gineityte, V. J Mol Struct (Theochem) 1995, 343, 183] and the well-known relation between the energy $E$ and the DM $P[E=\operatorname{Trace}(P H)$, where $H$ is the Hamiltonian matrix]. Inasmuch as the power series for the DM $P$ is based on a certain generalization of the usual Rayleigh-Schrödinger perturbation theory (RSPT), namely, on the so-called non-commutative RSPT [Gineityte, V. Int J Quantum Chem 1998, 68, 119], the new expression for total energies of molecules proved to be a generalization of the well-known Dewar formula obtained using the usual RSPT in the framework of the Hückel model. The generalization consists of passing to the case of zero-order resonance parameters between pairs of bonding BOs (BBOs) and/or of antibonding BOs (ABOs). Comparative analysis of the Dewar formula and of its generalized version was carried out, and some new interpretations of the former are suggested. In particular, the negative second-order correction within the Dewar energy is shown to be made up of a difference between the stabilization energy due to the formation of bond orders between BBOs and $\mathrm{ABOs}$ and the destabilization energy describing the intramolecular charge transfer. The case of alkanes, in general, and a model system of two interacting $\mathrm{C}-\mathrm{C}(\mathrm{C}-\mathrm{H})$ bonds, in particular, are discussed as examples. (C) 2000 John Wiley \& Sons, Inc. Int J Quant Chem 77: 534-543, 2000
\end{abstract}

Key words: the Dewar formula; total energies; additivity; saturated hydrocarbons; noncommutative Rayleigh-Schrödinger perturbation theory

\section{Introduction}

$\mathbf{P}$ erturbation theory (PT) is among the most powerful approximate methods in quantum mechanics [1-4]. In particular, the well-known Rayleigh-Schrödinger PT (RSPT) for eigenvalues and eigenfunctions of operators [1-3] and an alter- native PT based on the resolvent formalism [4] may be mentioned here.

Various forms of the perturbation theory are widely used in quantum chemistry as well. To obtain the many-electron wave functions of molecules, the so-called Møller-Plesset (MP) partitioning of the total Hamiltonian operator is now generally applied $[5,6]$. In our context, the MP partitioning for localized orbitals [7-9] and its generalization 
for any type of reference function [6] are noteworthy.

The self-consistent version of the RSPT (or, otherwise, the coupled RSPT) [10-13] forms the basis of the perturbed Hartree-Fock calculations. Self-consistent perturbative approaches for Green's functions [13] and for density matrices [14-16] instead of one-electron orbitals also have been developed.

The Hückel-type model Hamiltonian matrices of molecules may be diagonalized algebraically if the matrix form of the usual RSPT is applied [17-20]. It is noteworthy that perturbative approaches allow general results to be obtained for entire series and classes of molecules in the framework of the Hückel-type model. For example, algebraic expressions for various types of polarizabilities of conjugated hydrocarbons (atom-atom, atom-bond, etc.) have been derived [19-21]. Moreover, an important rule about alternating signs of atom-atomic polarizabilities along the chain has been established for alternant conjugated hydrocarbons [21].

The Dewar formula for total energies of molecules $[17,18,22,23]$ also is among the most popular direct results of the RSPT. Although this expression was originally intended for investigation of unsaturated hydrocarbons [22], it proved to be even more applicable to saturated molecules in the basis of bond orbitals (BOs). The point is that the mean value of the energy gap between the bonding $\mathrm{BOs}$ (BBOs) and antibonding $\mathrm{BOs}$ (ABOs) of these molecules always exceeds the average resonance parameter considerably [23].

From the Dewar formula derived in [22], it follows that the zero-order correction $E_{(0)}$ to the total energy $E$ contains a sum of energies of isolated bonds, while the first-order correction $E_{(1)}$ vanishes. The second-order correction $E_{(2)}$ always takes a negative value and, consequently, it describes the stabilization energy of the given molecule versus the relevant set of isolated bonds.

Furthermore, the second-order correction $E_{(2)}$ contains a sum of increments, each of them depending on the direct interaction (resonance parameter) between a $\mathrm{BBO}$ of a particular bond and an $\mathrm{ABO}$ of another bond (let us call them the intersubset resonance parameters). On the other hand, the intrasubset interactions (i.e., resonance parameters between two BBOs or those between two ABOs) do not contribute to the correction $E_{(2)}$, and these may be actually ignored when deriving the expression for stabilization energy of molecules $[17,18]$. It should be noted here that both intra- and inter- subset resonance parameters were assumed to be first-order terms when deriving the Dewar formula $[22,23]$.

The noncommutative version of the RSPT in the framework of the non-canonical MO (NCMO) method suggested recently [24-26] was shown to be a generalization [24] of the usual RSPT [1, 3]. In this connection, new possibilities for interpretation of the Dewar formula and for its generalization may be anticipated if we turn to this new perturbation theory.

In its matrix representation, the $\mathrm{NCMO}$ approach resolves itself into a block-diagonalization problem for the respective Fockian or Hückel-type Hamiltonian matrix [24-31]. Moreover, a block-diagonal zero-order matrix is allowed in this case [24-26].

It should be noted that diagonalility condition for zero-order matrices and / or operators is not imperative when applying perturbative approaches. Thus, in the resolvent-based PT [4], the zero-order operator is such that its eigenvalues and eigenfunctions can be easily determined. Similarly, the Fock operator of [7-9] is not necessarily diagonal in the orbital space. In this context, the distinctive feature of the noncommutative RSPT [24-26] consists of the feasibility of a general form of power series for entire blocks (submatrices) of both zero and firstorder Hamiltonian matrices whatever their actual dimensions and internal structures. Just the latter peculiarity of the noncommutative RSPT makes it possible to generalize the Dewar formula to the case of zero-order intrasubset resonance parameters.

An interest in the generalization of the abovementioned type originates from the results of estimations of relative values of intra- and intersubset resonance parameters. Thus, in the well-studied case of alkanes, the direct interactions between two nearest-neighboring BBOs exceed the intersubset resonance parameters considerably [32-34]. Such a conclusion may be traced back to the fact that resonance parameters between BOs of different types contain differences in the resonance parameters of separate pairs of $s p^{3}$-hybrid AOs (HAOs) and $1 s$ AOs, whereas those between two BBOs may be expressed as sums of four negative parameters in the $\mathrm{HAO}$ basis (BBOs and $\mathrm{ABO}$ are assumed to be defined as normalized sums and differences, respectively, of pairs of HAOs and 1s AOs belonging to the same bond).

An additional insight into the content of the Dewar formula itself is also likely to follow from a derivation of the total energy $E$ on the basis of the general expression for the one-electron den- 
sity matrix (DM) $P$ [26] instead of summing up the eigenvalues of the Hamiltonian matrix $H$ corresponding to occupied canonical MOs. This way of obtaining the total energy becomes feasible if we invoke the power series for the DM $P$ obtained in [26] and being actually based on the noncommutative RSPT [24, 25] and the well-known general relation between the total energy and the matrices $H$ and $P(E=\operatorname{Trace}(P H)[16])$. The reason why just the above-described way of derivation of the total energy seems to be worthwhile consists of the direct relation of the DM $P$ and, thereby, of the total en$\operatorname{ergy} E$ to particular blocks of the initial Hamiltonian matrix representing the intra- and intersubset interactions.

Therefore, the main aim of this article consisted of deriving and analyzing the generalized expression for total energies of molecules corresponding to zero-order intrasubset interactions. To this end, the power series for the DM $P$ obtained in [26] will be used. Thereupon, we will analyze the particular case of the first-order intrasubset interaction corresponding to the Dewar formula.

\section{The Generalized Expression for Total Energies}

Let us start with the Hückel-type model Hamiltonian matrix suggested in [26]. To construct this matrix, two bond orbitals were ascribed to each chemical bond in a molecule. These orbitals may be defined as eigenfunctions of separate twodimensional Hamiltonian matrix blocks associated with pairs of $s p^{3}$-hybrid AOs (HAOs) or of HAOs and $1 s$ AOs pertinent to the same bond. It should be noted, however, that the definition of BOs is not essential when deriving the general expression for the one-electron DM of saturated organic molecules. Hence, pairs of $2 p_{z}$ AOs of carbon atoms of the initially double bonds also may be used when defining BOs if we consider a conjugated compound. Orbitals referring to lone electron pairs (if any) also may be included into the initial basis set $[24,26]$. The whole set of basis functions $\{\phi\}$ has been assumed to be orthogonal. This set will be further referred to as the $\mathrm{BO}$ basis.

Let the first $n$ BOs $\phi_{(+) i}, i=1,2 \ldots n$ correspond to both the bonding BOs and the lone pair orbitals (if any), whereas the remaining BOs $\phi_{(-) i}$, $i=n+1, n+2 \ldots N$ will coincide with the antibonding BOs. Then, the matrix $H$ may be conveniently represented in terms of four submatrices (blocks) containing resonance parameters inside subspaces of $\mathrm{BBOs}$ and $\mathrm{ABOs}$ (the diagonal blocks) and those between $\mathrm{BOs}$ of different types (the off-diagonal blocks). First-order magnitude of the latter versus the differences between the $\mathrm{BBO}$ and $\mathrm{ABO}$ energies will be accepted [26]. This implies first-order magnitude of the off-diagonal blocks of the matrix $H$ (denoted by $R$ ) versus the diagonal ones. The abovementioned assumptions were borne out in [32-38] by the relevant estimations.

Let the diagonal blocks of the matrix $H$ be represented as sums of respective zero-order terms $E_{(+)}$ and $E_{(-)}$and of the respective first-order terms $T$ and $Q$, the subscripts " + " and "-" here and below referring to $\mathrm{BBOs} \phi_{(+) i}$ and $\mathrm{ABOs} \phi_{(-) i}$. Then, the total matrix $H$ takes the form of a sum of the zero-order and first-order Hamiltonian matrices:

$$
H=H_{(0)}+\lambda H_{(1)}
$$

where $\lambda$ is a formal perturbation parameter and

$$
H_{(0)}=\left|\begin{array}{cc}
E_{(+)} & 0 \\
0 & -E_{(-)}
\end{array}\right|, \quad H_{(1)}=\left|\begin{array}{cc}
T & R \\
R^{+} & Q
\end{array}\right| .
$$

The superscript " + " of the block $R$ designates the Hermitian-conjugate (transposed) matrix, and the minus sign in front of $E_{(-)}$is introduced for convenience. It is also seen that zero-order intrasubset resonance parameters are allowed within the Hamiltonian matrix of Eqs. (1) and (2), and these take the off-diagonal positions within the blocks $E_{(+)}$ and $E_{(-)}$.

The bond-order matrix $P$ corresponding to the Hamiltonian matrix $H$ of Eq. (1) was obtained in [26] in the form of the power series

$$
P=P_{(0)}+\lambda P_{(1)}+\lambda^{2} P_{(2)}+\cdots,
$$

and the first three terms of this series are

$$
\begin{aligned}
& P_{(0)}=\left|\begin{array}{cc}
2 I & 0 \\
0 & 0
\end{array}\right|, \quad P_{(1)}=-2\left|\begin{array}{cc}
0 & G_{(1)} \\
G_{(1)}^{+} & 0
\end{array}\right| \\
& P_{(2)}=-2\left|\begin{array}{cc}
G_{(1)} G_{(1)}^{+} & G_{(2)} \\
G_{(2)}^{+} & -G_{(1)}^{+} G_{(1)}
\end{array}\right| .
\end{aligned}
$$

(An idempotence requirement for the matrix $\frac{1}{2} P$ was among the initial conditions determining the matrix $P$ [26].) The principal matrices $G_{(1)}$ and $G_{(2)}$ of Eq. (4) meet the following equations:

$$
\begin{aligned}
& E_{(+)} G_{(1)}+G_{(1)} E_{(-)}+R=0 \\
& E_{(+)} G_{(2)}+G_{(2)} E_{(-)}+V=0,
\end{aligned}
$$

where

$$
V=T G_{(1)}-G_{(1)} Q .
$$


The relevant total energy may be defined as follows [16]:

$$
E=\operatorname{Tr}(P H),
$$

where $T r$ here and below stands for a Trace of matrix. After substituting Eqs. (1), (3), and (4) into Eq. (8), the expression for the total energy may also be obtained in the form of a power series:

$$
E=E_{(0)}+\lambda E_{(1)}+\lambda^{2} E_{(2)}+\cdots,
$$

where the first two corrections are

$$
\begin{aligned}
& E_{(0)}=\operatorname{Tr}\left(P_{(0)} H_{(0)}\right)=2 \operatorname{Tr} E_{(+)} \\
& E_{(1)}=\operatorname{Tr}\left(P_{(0)} H_{(1)}+P_{(1)} H_{(0)}\right)=2 \operatorname{Tr} T .
\end{aligned}
$$

The sum of these corrections coincides with the total one-electron energy of isolated BBOs. Given that the one-electron energy of any $\mathrm{BBO}$ is included into the respective diagonal element of the matrix $E_{(+)}$(this may be evidently done without any restriction), the first-order correction $E_{(1)}$ takes a zero value as it was established in $[22,23]$.

The second-order correction $E_{(2)}$ may be conveniently expressed in the form of a sum

$$
E_{(2)}=E_{(2)}^{\text {inter }}+E_{(2)}^{\text {intra }},
$$

where

$$
E_{(2)}^{\text {inter }}=\operatorname{Tr}\left(H_{(1)} P_{(1)}\right)=-2 \operatorname{Tr}\left(G_{(1)} R^{+}+G_{(1)}^{+} R\right)
$$

and

$$
\begin{aligned}
E_{(2)}^{\text {intra }} & =\operatorname{Tr}\left(P_{(2)} H_{(0)}\right) \\
& =-2 \operatorname{Tr}\left(G_{(1)} G_{(1)}^{+} E_{(+)}+G_{(1)}^{+} G_{(1)} E_{(-)}\right) .
\end{aligned}
$$

The first term of the sum $\left(E_{(2)}^{\text {inter }}\right)$ evidently describes the contribution of the intersubset bond orders represented by matrices $G_{(1)}$ and $G_{(1)}^{+}$to the total correction $E_{(2)}$, whereas the second term $\left(E_{(2)}^{\text {intra }}\right)$ contains the increment of the intrasubset bond orders along with populations of basis orbitals. This does not imply, however, that $E_{(2)}^{\text {inter }}$ is determined only by the intersubset resonance parameters and/or $E_{(2)}^{\text {intra }}$ is conditioned solely by intrasubset ones. Indeed, the principal matrix $G_{(1)}$ involved within both parts of the correction $E_{(2)}$ depends on both types of resonance parameters. [Note that three matrices $E_{(+)}, E_{(-)}$, and $R$ are contained within Eq. (5) determining the matrix $G_{(1)}$.]

Inasmuch as the diagonal blocks of the secondorder correction $P_{(2)}$ of the DM $P$ (intrasubset bond orders) are made up of the off-diagonal blocks $G_{(1)}$ and $G_{(1)}^{+}$of the relevant first-order correction $P_{(1)}$ (intersubset bond orders), an interdependence may be expected between the two parts of the total energy $E$.

To find this interrelation, let us use the cyclic transposition of matrices inside the Trace sign of Eq. (14) and invoke the Hermitian-conjugate counterpart of the relation shown in Eq. (5). We then obtain an expression for $E_{(2)}^{\text {intra }}$ in terms of intersubset bond orders, namely:

$$
E_{(2)}^{\text {intra }}=2 \operatorname{Tr}\left(G_{(1)} R^{+}\right) .
$$

On the other hand, from Eq. (13), a similar expression for $E_{(2)}^{\text {inter }}$ follows:

$$
E_{(2)}^{\text {inter }}=-4 \operatorname{Tr}\left(G_{(1)} R^{+}\right) .
$$

Comparison of Eqs. (15) and (16) shows that

$$
E_{(2)}^{\text {inter }}=-2 E_{(2)}^{\text {intra }} .
$$

As a result, the total correction $E_{(2)}$ is

$$
E_{(2)}=-E_{(2)}^{\text {intra }}
$$

or to

$$
E_{(2)}=\frac{1}{2} E_{(2)}^{i n t e r}
$$

for any molecule described by the Hamiltonian matrix $H$ of Eqs. (1) and (2). Equation (17) indicates that contributions of the intra- and intersubset bond orders to the total correction $E_{(2)}$ are of opposite signs. Moreover, the absolute value of the intersubset term exceeds twice the relevant value of the intrasubset contribution.

Substituting Eqs. (15) and (16) into Eq. (12) yields the following simple expression for the total correction $E_{(2)}$ :

$$
E_{(2)}=-2 \operatorname{Tr}\left(G_{(1)} R^{+}\right) .
$$

Equation (20) may be regarded as a generalization of the Dewar formula for total energies of molecules as discussed below. An alternative form of this expression is

$$
E_{(2)}=-2 \sum_{i, j} G_{(1) i j} R_{i j} .
$$

It is seen that the second-order correction to the total energy is additive with respect to contributions of pairs of bonds. (Note that any element $G_{(1) i j}$ may be ascribed to a pair of bonds $I$ and $J$ [26].) It should be emphasized, however, that the element $G_{(1) i j}$ does not describe a local interbond interaction in the general case in contrast to the resonance parameter $R_{i j}$. Indeed, the solution of Eq. (5) may be represented as an integral [24, 26, 39]:

$$
G_{(1)}=\int_{0}^{\infty} e^{E_{(+)} t} R e^{E_{(-)}} d t .
$$


It is seen that the element $G_{(1) i j}$, of the matrix $G_{(1)}$ depends on the whole set of elements of three matrices $\left(\exp \left[E_{(+)} t\right], \exp \left[E_{(-)} t\right]\right.$, and $\left.R\right)$, and the contribution $G_{(1) i j} R_{i j}$ of Eq. (21) ascribed to the pair of bonds $(I, J)$ is nonlocal in nature.

Using Eqs. (14) and (18), an alternative form of the total correction $E_{(2)}$ may be obtained, namely:

$$
E_{(2)}=2 \operatorname{Tr}\left(G_{(1)} G_{(1)}^{+} E_{(+)}+G_{(1)}^{+} G_{(1)} E_{(-)}\right) .
$$

This expression allows us to study the sign of the second-order term $E_{(2)}$ of the power series for the total energy $E$ as shown in the next section.

\section{Investigation of the Sign of the Second-order Energy}

Let us start with Eq. (23) and note that the Hermitian (symmetric) matrices $E_{(+)}$and $E_{(-)}$may be expressed in terms of diagonal matrices of eigenvalues $\left\|\epsilon_{(+) i}\right\|$ and $\left\|\epsilon_{(-) k}\right\|$ and the respective unitary (orthogonal) diagonalizing matrices $U$ and $W$ [39]:

$$
E_{(+)}=U^{+}\left\|\epsilon_{(+) i}\right\| U, \quad E_{(-)}=W^{+}\left\|\epsilon_{(-) k}\right\| W .
$$

Substituting Eq. (24) into Eq. (23) yields

$$
\begin{aligned}
E_{(2)}=2 \operatorname{Spur}\left\{G_{(1)} G_{(1)}^{+}\right. & U^{+}\left\|\epsilon_{(+) i}\right\| U \\
& \left.+G_{(1)}^{+} G_{(1)} W^{+}\left\|\epsilon_{(-) k}\right\| W\right\} .
\end{aligned}
$$

After a cyclic transposition of factors within the Trace sign of Eq. (25), the following expression for the correction $E_{(2)}$ results:

$$
\begin{aligned}
E_{(2)}=2 \sum_{i, j}\left\{\left(U G_{(1)}\right)_{i j}\right\}^{2} \epsilon_{(+) i} & \\
& +2 \sum_{l, k}\left\{\left(W G_{(1)}^{+}\right)_{l k}\right\}^{2} \epsilon_{(-) k} .
\end{aligned}
$$

It is seen that negative values of both $\epsilon_{(+) i}$ and $\epsilon_{(-) k}$ are sufficient for a negative value of the total correction $E_{(2)}$. Thus, the second-order correction yields stabilization of the system versus the set of isolated bonds if $E_{(+)}$and $E_{(-)}$are negative-definite matrices [39, 40]. Otherwise, the sign of the correction $E_{(2)}$ is not evident a priori.

It may be concluded, therefore, that signs of eigenvalues of matrices $E_{(+)}$and $E_{(-)}$serve as a criterion for stabilization (or destabilization) of the system versus the respective set of isolated bonds. This does not imply, of course, that only the intrasubset resonance parameters contained within these matrices determine the sign of the second-order energy $E_{(2)}$. The above-established relation between the sign of the correction $E_{(2)}$ and those of eigenvalues of matrices $E_{(+)}$and $E_{(-)}$may be accounted for by interdependence between the intra- and intersubset contributions of this correction and, thereby, by the possibility of expressing the total correction second-order energy $E_{(2)}$ via its intrasubset part as shown in Eqs. (18) and (23).

To illustrate the above conclusions, let us turn again to the case of alkanes $[23,26,30,31,35-$ $37,41-45]$. One-electron energies of BBOs of these molecules represented by diagonal elements of matrices $E_{(+)}$are known to be of similar values for $C-C$ and $\mathrm{C}-\mathrm{H}$ bonds [41-43], and the same is likely to be true for $\mathrm{ABOs}$ as well. If the energy reference point is chosen in the middle of the energy gap between BBOs and $\mathrm{ABOs}$, the diagonal elements of both matrices $E_{(+)}$and $E_{(-)}$take negative values. [The minus sign in front of $E_{(-)}$within Eq. (2) also should be taken into consideration.] Then, $E_{(+)}$and $E_{(-)}$may be expected to be negative-definite matrices if their off-diagonal elements (intrasubset resonance parameters) are not too large as compared to the above-mentioned energy gap and thereby their eigenvalues also are negative.

The off-diagonal elements of matrices $E_{(-)}$of alkanes prove to be small because of different signs of their four components in the HAO basis. By contrast, the resonance parameters between two nearest-neighboring (geminal) BBOs are of considerable values approximately equal to $\beta \simeq-3 \mathrm{eV}$ [41-43]. In this connection, the highest eigenvalues of matrices $E_{(+)}$should be compared to the energy reference point to establish the signs of former.

The energy gap between BBOs and ABOs, in turn, is determined by the double mean value of resonance parameters for pairs of HAOs and Is AOs belonging to the same $\mathrm{C}-\mathrm{C}(\mathrm{C}-\mathrm{H})$ bond. The averaged value of these resonance parameters has been established to be equal to $-6.7 \mathrm{eV}$ [44]. Hence, the diagonal elements of matrices $E_{(+)}$of alkanes should be taken equal to $-6.7 \mathrm{eV}$ with respect to the abovedefined energy reference point.

Analysis of spectra of matrices $E_{(+)}$of alkanes carried out in $[45,46]$ showed that the abovementioned highest eigenvalue does not exceed $-2 \beta \simeq 6 \mathrm{eV}$ with respect to diagonal elements of these matrices. Hence, all the eigenvalues of matrices $E_{(+)}$may be assumed to be negative, if we revert to the former energy reference point. This conclusion is in line with the observed stability of alkanes (the experimental heats of formation may be found in [47]). Furthermore, the results of this section yield a more profound accounting for stability of alkanes. 
Indeed, the usual explanation of this fact consists in an assumption that resonance parameters between pairs of BBOs are sufficiently small and thereby the Dewar formula is adequate $[17,18$, 23]. The results of the above-discussed evaluations, however, make us question this point of view. This implies that peculiarities of the spectra of matrices $E_{(+)}$should be considered when discussing the origin of the stability of alkanes.

In this context, the whole spectra of matrices $E_{(+)}$(and not only their highest eigenvalues) are of interest. Comparison of the approximate limits to location of the highest eigenvalue $(-2 \beta)$ and of the lowest one (equal to $5 \beta \simeq-15 \mathrm{eV}$ ) to diagonal elements of matrices $E_{(+)}$indicates an asymmetric nature of these spectra. Just this asymmetry is able to ensure a negative sign of the second-order energy $E_{(2)}$ even if the off-diagonal elements of matrices $E_{(+)}$ (intrasubset resonance parameters) are sufficiently large.

Constitution of matrices $E_{(+)}$determining their spectra and thereby conditioning the above-mentioned asymmetry depends on spacial arrangement of $\mathrm{BOs}$ and thereby of bonds. Therefore, the tetrahedral spatial arrangement of quartets of bonds at the same carbon atom (molecular topology) may be considered as the main origin of stability of alkanes versus the respective sets of isolated bonds.

\section{The Particular Case of the First-order Intrasubset Interaction}

The Dewar case follows from the relations of the second section if $E_{(+)}$and $E_{(-)}$are diagonal matrices containing elements $E_{(+) i}$ and $E_{(-) j}$. Then, the elements $G_{(1) i j}$ of the principal matrix $G_{(1)}$ take the form [26]

$$
G_{(1) i j}=-\frac{R_{i j}}{E_{(+) i}+E_{(-) j}}
$$

and describe the direct (through-space) interactions between BBOs and ABOs $\left[\phi_{(+) i}\right.$ and $\left.\phi_{(-) j}\right]$. If we substitute Eq. (27) into Eq. (21), the Dewar formula for the second-order correction to the total energy follows $[17,18,22,23]$ :

$$
E_{(2)}^{(D)}=2 \sum_{i, j} \frac{\left(R_{i j}\right)^{2}}{E_{(+) i}+E_{(-) j}}<0 .
$$

The negative sign of the Dewar energy is obvious as $E_{(+) i}<0$ and $E_{(-) j}<0$.
Derivation of the total energy on the basis of the DM $P$ as discussed in the second section allows also certain conclusions to be drawn about the internal constitution of $E_{(2)}^{(D)}$. Indeed, the relations shown in Eqs. (12) and (17)-(19) hold true for the Dewar case as well. Using Eqs. (16) and (27), the intersubset part of the Dewar energy may be expressed in the form

$$
E_{(2)}^{(D) i n t e r}=4 \sum_{i, j} \frac{\left(R_{i j}\right)^{2}}{E_{(+) i}+E_{(-) j}}<0 .
$$

This contribution describes the total stabilizing effect of the intersubset bond orders arising between $\mathrm{BBOs}$ and ABOs. It is evident that this effect exceeds twice the final second-order correction $E_{(2)}^{(D)}$ shown in Eq. (28). Hence, the remaining part of the Dewar energy $\left[E_{(2)}^{(D) i n t r a}\right]$ may be expected to represent certain destabilization of the system. The latter effect proves to be related to the intramolecular charge transfer.

To demonstrate this, let us define the following matrices:

$$
Q_{(+)}=-2 G_{(1)} G_{(1)}^{+}, \quad Q_{(-)}=2 G_{(1)}^{+} G_{(1)} .
$$

From Eq. (4), it follows that diagonal elements of these matrices describe alterations in occupation numbers of BOs after building up the molecule. Thus, $Q_{(+) i}$ represents the population lost by the initially double-occupied $\mathrm{BBO} \phi_{(+) i}$, while $Q_{(-) i}$ coincides with the population acquired by the initially vacant $\mathrm{ABO} \phi_{(-) i}$, and $Q_{(+) i}<0$ while $Q_{(-) i}>0$. Substituting Eq. (30) into Eq. (14) yields the intrasubset contribution to the Dewar energy:

$$
E_{(2)}^{(D) \text { intra }}=\sum_{i \in I}^{\text {bonds }}\left(Q_{(+) i} E_{(+) i}-Q_{(-) i} E_{(-) i}\right)>0,
$$

which may be alternatively called the chargetransfer energy. The positive sign of $E_{(2)}^{(D) \text { intra }}$ may be accounted for by the fact that charge is being transferred from the orbitals of the lower energy (BBOs) to those of the higher energy (ABOs) when building up the molecule, and this effect causes certain destabilization of the system. Therefore, the final second-order correction of the Dewar formula $E_{(2)}^{(D)}$ actually describes a difference between the stabilization energy due to the formation of the intersubset bond orders $\left[E_{(2)}^{(D) i n t e r}\right]$ and the destabilization energy being related to the intramolecular charge transfer $\left[E_{(2)}^{(D) \text { intra }}\right]$.

From Eq. (31) it is seen that $E_{(2)}^{(D) \text { intra }}$ is additive with respect to contributions of separate bonds. If we invoke Eq. (18) in addition, the total correction 
$E_{(2)}^{(D)}$ also may be represented in an additive form, namely:

$$
E_{(2)}^{(D)}=-\sum_{i \in I}^{\text {bonds }}\left(Q_{(+) i} E_{(+) i}-Q_{(-) i} E_{(-) i}\right) .
$$

Therefore, the Dewar energy $E_{(2)}^{(D)}$ proves to be additive with respect to increments of separate bonds whatever the approximations used within the initial Hamiltonian matrix. In [23], an analogous additivity of the energy $E_{(2)}^{(D)}$ was established provided that resonance parameters $R_{i j}$ between the nearestneighboring (geminal) bonds only are taken into consideration. It should be also mentioned here that the extents of transferability of increments of particular bonds to the energy $E_{(2)}^{(D)}$ are evidently related to those of occupation numbers of BOs.

In $[26,31]$, the population alterations $Q_{(+) i}$ and $Q_{(-) i}$ were related to total delocalization coefficients $D_{(+) i}$ and $D_{(-) i}$ of noncanonical MOs (NCMOs) $\Psi_{(+) i}$ and $\Psi_{(-) i}$, corresponding to BOs $\phi_{(+) i}$ and $\phi_{(-) i}$, respectively. (Correspondence implies here that just this $\mathrm{BO}$ makes the principal contribution to the NCMO under discussion.) The coefficients $D_{(+) i}$ and $D_{(-) i}$ describe the extents of delocalization of NCMOs $\Psi_{(+) i}$ and $\Psi_{(-) i}$ versus the respective BOs and the above-mentioned relations take the form

$$
Q_{(+) i}=-2 D_{(+) i}, \quad Q_{(-) i}=2 D_{(-) i} .
$$

If we substitute Eq. (33) into Eq. (32), an expression for the energy $E_{(2)}^{(D)}$ in terms of delocalization coefficients of NCMOs follows, namely:

$$
E_{(2)}^{(D)}=2 \sum_{i \in I}^{\text {bonds }}\left(D_{(+) i} E_{(+) i}+D_{(+) i} E_{(+) i}\right) .
$$

Therefore, stabilization of the molecule versus the set of isolated bonds may be concluded to be directly related to the extent of interbond delocalization.

Before finishing this section, let us revert again to the general form of the second-order correction to the total energy corresponding to the zero-order intrasubset interaction $\left[E_{(2)}\right]$ and shown in Eqs. (20) and (23). Two points may be mentioned here:

First, the correction $E_{(2)}$ may be represented as a sum of the Dewar energy $E_{(2)}^{(D)}$ and of an additional increment of the intrasubset bond orders $Q_{(+) i j}$ and $Q_{(-) i j}(i \neq j)$. Indeed, using Eqs. (23) and (30), the energy $E_{(2)}$ may be represented in the form

$$
E_{(2)}=-\operatorname{Spur}\left(Q_{(+)} E_{(+)}-Q_{(-)} E_{(-)}\right) .
$$

On the basis of Eqs. (32) and (35), we then obtain

$$
E_{(2)}=E_{(2)}^{(D)}-\sum_{i \neq j}\left(Q_{(+) i j} E_{(+) i j}-Q_{(-) i j} E_{(-) i j}\right) .
$$

The increment of the intrasubset bond orders represented by the second term of the right side of Eq. (36) evidently turns to zero if the zero-order intrasubset resonance parameters $E_{(+) i j}$ and $E_{(-) i j}$ $(i \neq j)$ vanish. In the general case, it is this increment that is able to cause destabilization of the system.

Second, a self-consistent version of Eq. (20) also seems to be feasible. The power series for the DM $P$ underlying Eq. (20) and shown in Eq. (3) may be obtained either using the solutions of the blockdiagonalization problem [25] originating from the Brillouin theorem [24-30] or from a direct solution of the commutation equation for matrices $H$ and $P$ [26]. Thus, the self-consistent analog of Eq. (20) is likely to be obtainable on the basis of both the known self-consistent versions of the abovementioned two matrix problems [16, 48, 49] and the experience of deriving the self-consistent version of the Dewar formula. (The latter evidently coincides with the second-order correction for the total energy within the coupled RSPT [10-13].)

\section{Comparison of Relative Accuracies of the Dewar Formula and of Its Generalized Version: A Model System of Two Interacting Bonds}

A simple model system containing two interacting bonds served many times as an illustrative example when studying electronic structures of both alkanes and their derivatives [33, 35, 38, 50]. Thus, we will invoke this model for comparison of relative accuracies of Eqs. (20) and (28).

Let $\gamma$ and $\delta$ stand for resonance parameters describing the interaction between the two BBOs $\phi_{(+) 1}$ and $\phi_{(+) 2}$ of the two-bond system and that between a $\mathrm{BBO}$ and an $\mathrm{ABO}$, respectively. The interaction between ABOs $\phi_{(-) 1}$ and $\phi_{(-) 2}$ is assumed to take a zero value for simplicity of the model. The formal parameter $\lambda$ of Eq. (1) is supposed to be incorporated into parameters $\gamma$ and $\delta$ so that the equality $\gamma=1$ may be accepted. Finally, the energy unit will coincide with the averaged resonance parameter between HAOs belonging to the same bond.

Let us start with the Dewar case. Then, both, $\gamma$ and $\delta$ will be considered as first-order terms versus the energy difference between BBOs and ABOs 
equal to 2. As a result, the submatrices of Eq. (2) take the form

$$
\begin{aligned}
& E_{(+)}=E_{(-)}=I, \quad T=\left|\begin{array}{ll}
0 & \gamma \\
\gamma & 0
\end{array}\right|, \\
& Q=0, \quad R=\left|\begin{array}{ll}
0 & \delta \\
\delta & 0
\end{array}\right| .
\end{aligned}
$$

Application of Eqs. (10), (11), and (28) yields an expression

$$
E^{(D)}=4+2 \delta^{2},
$$

where 4 corresponds to the zero-order energy. It is seen that the intrasubspace interaction $\gamma$ does not contribute to the Dewar energy in accordance with the expectation $[17,18]$.

Let us turn now to the generalized approximation for total energies shown in Eq. (20). Application of this formula implies that the parameter $\gamma$ is included into the zero-order submatrix $E_{(+)}$, that is:

$$
\begin{aligned}
& E_{(+)}=\left|\begin{array}{ll}
1 & \gamma \\
\gamma & 1
\end{array}\right|, \quad E_{(-)}=I, \\
& T=Q=0, \quad R=\left|\begin{array}{ll}
0 & \delta \\
\delta & 0
\end{array}\right| .
\end{aligned}
$$

To find the relevant matrix $G_{(1)}$, the equation of Eq. (5) should be solved for submatrices $E_{(+)}, E_{(-)}$, and $R$ taken from Eq. (39). We then obtain

$$
G_{(1)}=\frac{\delta}{4-\gamma^{2}}\left|\begin{array}{cc}
\gamma & -2 \\
-2 & \gamma
\end{array}\right|
$$

and the new expression for total energies of two interacting bonds takes the form

$$
E^{(N)}=4+\frac{8 \delta^{2}}{4-\gamma^{2}} .
$$

It is seen that both $\delta$ and $\gamma$ determine the interbond interaction energy in this case, in contrast to Eq. (38). Moreover, the new formula turns into Eq. (38) if the equality $\gamma=0$ is assumed. This result promotes an expectation that the relative accuracy of Eqs. (38) and (41) depends on the actual value of $\gamma$ as compared to $\delta$.

To verify this expectation, the expression for the exact energy of two interacting bonds also is required. The latter follows after summing up the two highest eigenvalues of the total Hamiltonian matrix

$$
H=\left|\begin{array}{cccc}
1 & \gamma & 0 & \delta \\
\gamma & 1 & \delta & 0 \\
0 & \delta & -1 & 0 \\
\delta & 0 & 0 & -1
\end{array}\right|
$$

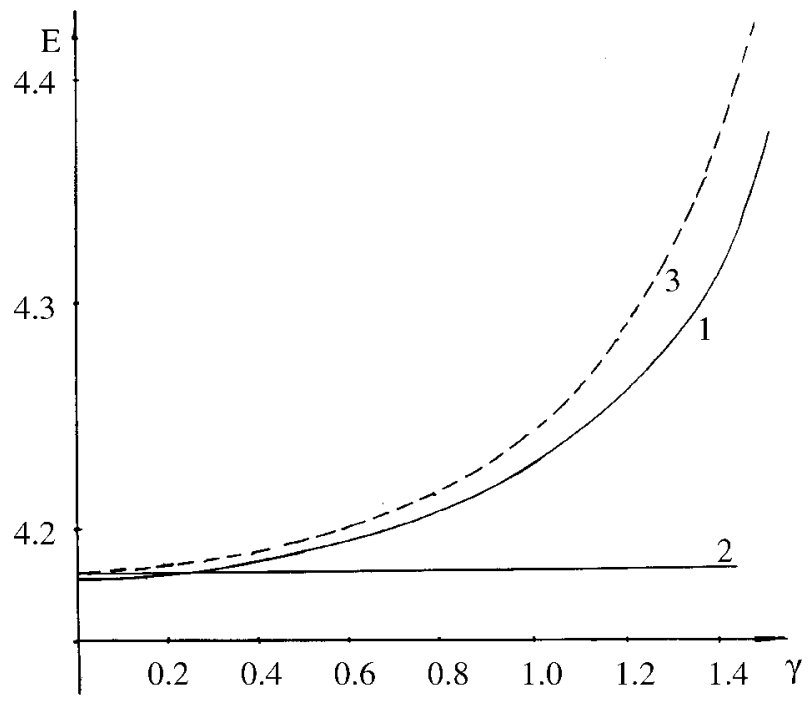

FIGURE 1. Dependence of the total energy of two interacting bonds $(E)$ on the resonance parameter $\gamma$ describing the interaction between the two BBOs. The resonance parameter $\delta$ representing an interaction between a BBO and an $\mathrm{ABO}$ is taken equal to 0.3. The exact expression for the energy defined by Eq. (43) is displayed by curve 1 , whereas the approximate formulas are represented by curves 2 and 3: The former corresponds to the Dewar formula [Eq. (28)] and the latter describes its generalized version suggested in this article [(Eq. (20)].

obtained without invoking any power series. Thus, the exact interbond interaction energy takes the form

$$
E=\sqrt{\gamma^{2}+4\left(1+\gamma+\delta^{2}\right)}+\sqrt{\gamma^{2}+4\left(1-\gamma+\delta^{2}\right)} .
$$

The dependencies of $E^{(D)}, E^{(N)}$, and $E$ on resonance parameter $\gamma$ are displayed in Figure 1. It is seen that the Dewar approximation is more accurate for small $\gamma$ values $(0<\gamma<0.4)$ being comparable to the $\delta$ value $(\delta=0.3)$. This result evidently causes no surprise. For large $\gamma$ values, however, the generalized formula of Eq. (20) becomes considerably more accurate. Moreover, the total interval of $\gamma$ values, wherein the approximation of Eq. (41) is valid, exceeds the relevant region of adequacy of the Dewar formula, and it actually includes all the reasonable $\gamma$ values. Therefore, the validity of Eq. (20) for large intrasubset resonance parameters as compared to the intersubset ones is beyond any doubt. 


\section{Conclusions}

Application of the noncommutative RSPT allowed us to generalize the Dewar formula for total energies of molecules to the case of zero-order intrasubset resonance parameters. It is noteworthy that some essential features of the Dewar formula are inherent in the generalized expression as well, namely, the zero value of the first-order correction $E_{(1)}$ and additivity of the second-order correction $E_{(2)}$ with respect to increments of separate pairs of bonds.

Again, the general case proves to be characterized by a nonlocal nature of increments of bond pairs in contrast to the Dewar formula. Moreover, the sign of the second-order correction $E_{(2)}$ is not defined a priori, whereas the relevant correction of the Dewar expression is known to be negative. The sign of the generalized correction $E_{(2)}$ proved to be determined by both the relative values of the intrasubset resonance parameters versus the energy gap between $\mathrm{BBO}$ and $\mathrm{ABO}$ and by the spatial arrangement of bonds (molecular topology) of molecules under study.

Certain conclusions about the content of the Dewar formula itself also may be drawn on the basis of the present study. Thus, the negative second-order correction $E_{(2)}^{(D)}$ describing the stabilization energy of a molecule versus the relevant set of isolated bonds is actually made up of a difference between the stabilization energy due to intersubset bond orders and the destabilization energy related to the intramolecular charge transfer. Furthermore, the absolute value of the former contribution exceeds twice the relevant value of the latter. Also, finally, the secondorder correction $E_{(2)}^{(D)}$ within the Dewar formula may be alternatively interpreted as the interbond delocalization energy. Additivity of the correction $E_{(2)}^{(D)}$ with respect to increments of separate bonds was established whatever the approximations used within the initial Hamiltonian matrix.

\section{References}

1. Landau, L. D.; Lifshits, E. M. Quantum Mechanics. The Nonrelativistic Theory; Nauka: Moscow, 1974.

2. Zülicke, L. Quantenchemie, Bd.1, Grundlagen und Algemeine Methoden; Deutscher Verlag der Wissenschaften: Berlin, 1973.

3. Flygare, W. H. Molecular Structure and Dynamics; PrenticeHall: Englewood Cliffs, NJ, 1978.
4. Economou, E. N. Green's Functions in Quantum Physics; Springer-Verlag: Berlin, Heidelberg, New York, 1979.

5. Wolinsky, K.; Pulay, P. J Chem Phys 1989, 90, 3647.

6. Wolinsky, K.; Sellers, H. L.; Pulay, P. Chem Phys Lett 1987, 140, 225.

7. Pulay, P.; Saebo, S. Theor Chim Acta 1986, 69, 357.

8. Saebo, S.; Pulay, P. J Chem Phys 1987, 86, 914.

9. Saebo, S.; Pulay, P. J Chem Phys 1988, 88, 1884.

10. Stevens, R. M.; Pitzer, R. M.; Lipscomb, W. N. J Chem Phys 1963, 38, 550 .

11. Malychanov, J. B. Zh Strukt Khim 1982, 23, 134.

12. Caves, T. C.; Karplus, M. J Chem Phys 1969, 50, 3649.

13. McDowell, H. K.; Porter, R. N. J Chem Phys 1976, 65, 4210.

14. Dierksen, O.; McWeeny, R. J Chem Phys 1966, 44, 355.

15. Dodds, J. L. N.; McWeeny, R. Mol Phys 1977, 33, 611.

16. Mestetchkin, M. M. The Density Matrix Method in Quantum Chemistry; Naukova Dumka: Kiev, 1977 (in Russian).

17. Dewar, M. J. S.; Dougherty, R. C. The PMO Theory of Organic Chemistry; Plenum: New York, 1975.

18. Dewar, M. J. S. The Molecular Orbital Theory of Organic Chemistry; McGraw-Hill: New York, 1969.

19. Zahradnik, R.; Polák, R. Elements of Quantum Chemistry; Plenum: New York, 1980.

20. Basilevskii, V. M.; The MO Method and Reactivity of Organic Molecules; Khimia: Moscow, 1969.

21. Coulson, C. A.; Longuet-Higgins, H. C. Proc R Soc A 1947, 191, 39; 1947, 192, 16; 1948, 193, 447, 456; 1948, 195, 188.

22. Dewar, M. J. S.; Pettit, R. J Chem Soc 1954, 1625.

23. Dewar, M. J. S. J Am Chem Soc 1984, 106, 669.

24. Gineityte, V. Int J Quantum Chem 1998, 68, 119.

25. Gineityte, V. Int J Quantum Chem 1999, 72, 559.

26. Gineityte, V. J Mol Struct (Theochem) 1995, 343, 183.

27. Mayer, I. Chem Phys Lett 1982, 89, 390.

28. Surján, P. R.; Mayer, I.; Kertész, M. J Chem Phys 1982, 77, 2454.

29. Mayer, I.; Surján, P. R. J Chem Phys 1984, 80, 5649.

30. Gineityte, V. J Mol Struct (Theochem) 1993, 288, 111.

31. Gineityte, V. J Mol Struct (Theochem) 1995, 333, 297.

32. Shatkovskaya, D.; Gineityte, V.; Bolotin, A. B. Theor Eksper Khim 1986, 2, 168.

33. Gineityte, V.; Shatkovskaya, D. Zh Strukt Khim 1984, 25, 152.

34. Satkovskiene, D.; Gineityte, V. Int J Quantum Chem 1996, 58, 453.

35. Pople, J. A.; Santry, D. P. Mol Phys 1963-64, 7, 269.

36. Gineityte, V.; Shatkovskaya, D. J Mol Struct (Theochem) 1989, 201, 49.

37. Gineityte, V.; Shatkovskaya, D. Int J Quantum Chem 1991, $39,11$.

38. Gineityte, V.; Shatkovskaya, D. Zh Strukt Khim 1985, 26, 42.

39. Lankaster, P. Theory of Matrices; Academic: New York, 1969.

40. Strang, G. Linear Algebra and Its Applications; Academic: New York, 1976.

41. Bieri, G.; Dill, J. D.; Heilbronner, E.; Schmelzer, A. Helv Chem Acta 1977, 60, 2234.

42. Heilbronner, E. Helv Chem Acta 1977, 60, 2248. 


\section{DEWAR'S FORMULA FOR TOTAL ENERGIES OF MOLECULES}

43. Heilbronner, E. In The Chemistry of Alkanes and Cycloalkanes; Patai S.; Rappoport Z., Eds.; Wiley: New York, 1992; pp. 455-529.

44. Gineityte, V. J Mol Struct (Theochem) 1998, 430, 97.

45. Gineityte, V. Lith J Phys 1992, 32, 528 (Russian Ed.); 1992, 32, 277 (Engl. Ed.).
46. Gineityte, V. Int J Quantum Chem 1997, 64, 481.

47. Weast, R. C. RCR Handbook of Chemistry and Physics; CRC: Boca Raton, FL, 1986.

48. Lefebvre, R. Comp Rend Acad Sci 1955, 240, 1094.

49. Daudey, J. P. Chem Phys Lett 1974, 24, 574.

50. Murrell, J. N.; Gil, V. M. S. Theor Chim Acta 1966, 4, 114. 